\title{
FIRE HAZARDS IN LITHIUM-ION BATTERIES UNDER OVERCHARGING AND SAFETY STRATEGIES
}

\author{
M Prithviraj \\ Department of Health Safety and Environment \\ University of Petroleum and Energy Studies, Dehradun, Uttarakhand, India
}

\begin{abstract}
Various Li-ion battery (LIB) fire and blasts have raised real worries about the safety issues connected with LIBs, some of those occurrences were in the main brought about by overcharging of LIBs. Thus, to possess a superior comprehension of the fire dangers brought about by LIB overcharging, 2 broadly utilised industrial LIBs, nickel manganese cobalt oxide (NMC) and lithium iron phosphate (LFP), were tried during this work. a couple of boundaries together with the surface temperature, the flame temperature, voltage, and radiative heat flux were calculable and investigated. The outcomes demonstrate that the underlying cathartic voltage increments with the event of charge cut-off voltage. AN overcharged LIB can undergo a additional vicious burning cycle and has lower steadiness than a typical one, and therefore the increasing cut-off voltage bothers the seriousness. Furthermore, this work examines the thermal runaway mechanism and presents completely different thermal runaway mitigation process, together with separators, flame retardants, and safety vents. The work then outlines measures for stifling Fires, and lands up with a bunch of suggestions for future innovative work.

Quercetin, an organic antioxidant, has been utilized as an additive in Li-ion cells to boost the chemistry performance to enhance the cycle life and also the overcharging characteristics. The quercetin -containing lithium battery showed an improvement in its electrochemical properties with $92 \%$ capacity retention after 350 cycles. the advance would possibly result from the formation of a passivation microstructure (from quercetin oxidation) on the electrode's surface. The quercetin-containing batteries provided long term cycling and a high safety performance, creating them a viable power supply for applications involving electrical devices with important safety requirements.
\end{abstract}

\section{INTRODUCTION}

Safety of lithium-based batteries has attracted much media and legal attention. Any energy storage device carries a risk. Lithium-ion is safe however with millions of consumers using batteries, disasters are sure to happen. In 2006, a one-in-
200,000 breakdown caused a recall of almost six million lithium-ion packs. Li-ion using traditional metallic oxides is nearing its theoretical limit on specific energy. Rather than optimizing ability, battery makers are enhancing production strategies to enhance protection and boom calendar life. The actual trouble lies while on rare events an electrical short develops in the cell. The outside safety peripherals are useless to forestall a thermal runaway as soon as in progress. Heat mixed with a full charge is stated to set off extra strain to Liion than regular cycling. Keep the battery and a device away from sun exposure and keep in a cool location at a partial charge. Exceeding the endorsed charge current by ultra-rapid changing also harms Li-ion. With the continuation of environmental issues together with global warming, greenhouse gas emissions, and sea-level rise due to significant use of fossil fuels, LIBs had been broadly used as a power source for electronic devices including laptops, cell phones, electric vehicles (EV), and hybrid electric vehicles (HEV) because of their excessive energy density, stable overall performance, lengthy life, and other excellent properties. However, Fires and explosions due to LIBs have frequently been reported. These incidents have raised true concerns about the protection of LIBs. In this manner, it's miles vital to direct in addition research at the fire risks of LIBs with the intention that we are able to make use of them better. In this work, we investigated the fire risks of extensively applied business LIBs, NMC and LFP, under overcharging conditions. Specific limitations along with cell voltage, floor temperature, flame temperature, and heat flux have been estimated. The effects have been almost dissected to offer vital statistics on execution based fire protection plan. In standard $\mathrm{Li}$-ion batteries with fluid electrolytes, there are five key segments: anode, cathode, separator, current collector, and electrolyte. Among those segments, the separator and the electrolyte are much less lenient to increasing temperature than the anodes and current collectors, that are manufactured from metallic oxide/graphite or metal. A Li-ion battery makes use of a polymer separator and a flammable electrolyte, that are each obliged to positive temperature limits for secure execution. At the point when a Li-ion battery's temperature increments to kind of $130-150{ }^{\circ} \mathrm{C}$, the excessive-energy substances and the herbal segments aren't constant and are willing to supply extra warmth. On the off 
chance that the created warmth does not disperse, the battery temperature will moreover increment and accelerate the warmth liberating process. Thermal runaway is probably spark off if a battery has positive affects that may activate shortcircuiting, is overheated, is susceptible to high pulse power use, or is penetrated. By and huge, the passivation layer (robust electrolyte interphase, SEI) at the cathode deteriorates at round $69{ }^{\circ} \mathrm{C}$. After the breakdown of the SEI layer, the electrolyte responds with the terminal and can provide flammable hydrocarbon gases. The polymer separator liquefies while the temperature is round $130{ }^{\circ} \mathrm{C}$. At better temperatures, the positive cathode decays and delivers oxygen. One of the issues hindering the economic utility of large capacity lithiumion batteries is the safety concern associated with them. Explosive accidents, and fire associated incidents, regarding Li-ion batteries are nevertheless often recorded; however, maximum incidents seem to contain over charging or overheating. In maximum instances the standard liquid electrolyte solutions used for lithium-ion batteries are natural solutions. However, those liquid electrolytes are easily flammable. The temperature and the internal pressure of the battery boom with an increase in inner impedance in the course of overcharging, eventually ensuing in fire and explosion. The protection trouble of lithium ion battery is particularly contributed via way of means of thermal runaway precipitated fire and explosion. The reason of this overview is to record the state of the art on lithium ion battery protection and associated thermal runaway prevention strategies and a way to enhance the battery protection or a way to prevent the thermal runaway. Safety is an vital trouble for lithium-ion batteries while they're overcharged. It is widely recognized that the addition of 'additive packages' to electrolytes will regulate their electrochemical overall performance. Several sorts of components had been proposed to enhance the cell's overcharging performance and flame retarding ability. The use of redox shuttle additives appears to be an powerful manner to enhance overcharging performance. A redox shuttle is an electrolyte additive that may be reversibly oxidized-decreased at a characteristic potential to provide an intrinsic overcharge safety for lithium-ion batteries. To enhance the thermal protection and the cycling performance of lithium-ion batteries, numerous fire retardant organic phosphates had been investigated. However, the upgrades acquired with respect to electrolyte protection in phrases of flame retardation and overcharging protecting are usually accompanied via way of means of degradation of the cell's key performance indicators together with its ability, because of changes withinside the electrolyte's various viscosity and ionic conductivity.

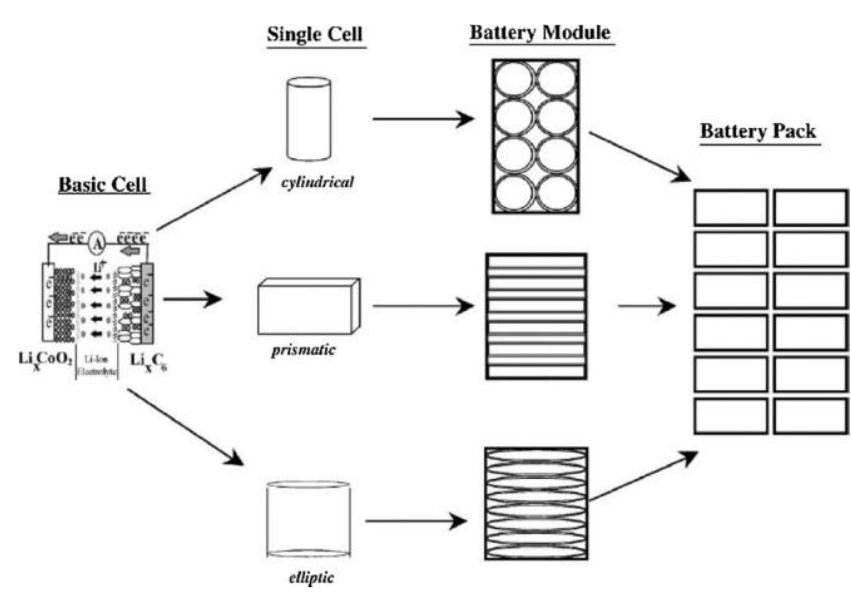

Figure 1. Schematics of the battery pack design with different cell configurations.

\section{OVERVIEW}

\subsection{Thermal runaway process}

This segment talks about how thermal runaway influences battery conduct. The thermal runaway measure influences the battery voltage, temperature, and pressure. The battery drops pointedly before thermal runaway because of the lamination of the battery terminals. The battery temperature increments in light of the very fact that the heat generation rate overpowers the heat dissipating rate. Moreover, because of the responses among battery dynamic materials, natural solution dissipation and gas age cause an amassing of gases within the battery. below normal activity conditions, the battery open circuit voltage (OCV) doesn't drop pointedly, although a pointy voltage drop shows the deficiency of battery trait and inner short out. Thermal runaway is one among the failure modes in batteries. many researchers have been conducted to search out the precise reason for this issue and the way to stop it. Generally, thermal runaway happens once an reaction goes out of management that is the reaction rate will increase because of a rise in temperature inflicting an additional increase in temperature and thus a further increase within the reaction rate, that presumably leading to an explosion. it's projected that higher than $80{ }^{\circ} \mathrm{C}$, thermal runaway will occur spontaneously as a results of fire or explosion. For the lithium ion battery runaway it's caused by the heat-releasing reactions between the electrolyte, anode and cathode, with the temperature and pressure increasing within the battery, the battery can rupture at last. 


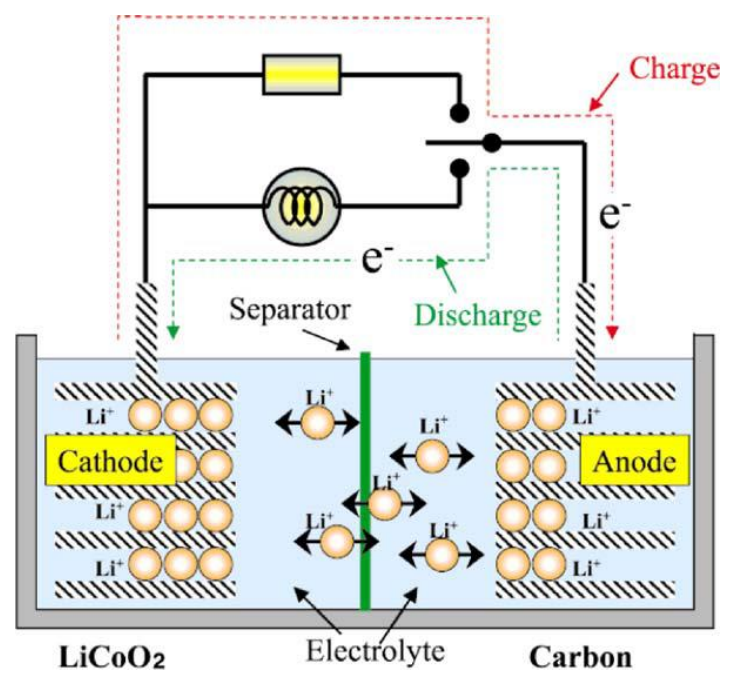

Figure 2. Schematic of the principle of LIB.

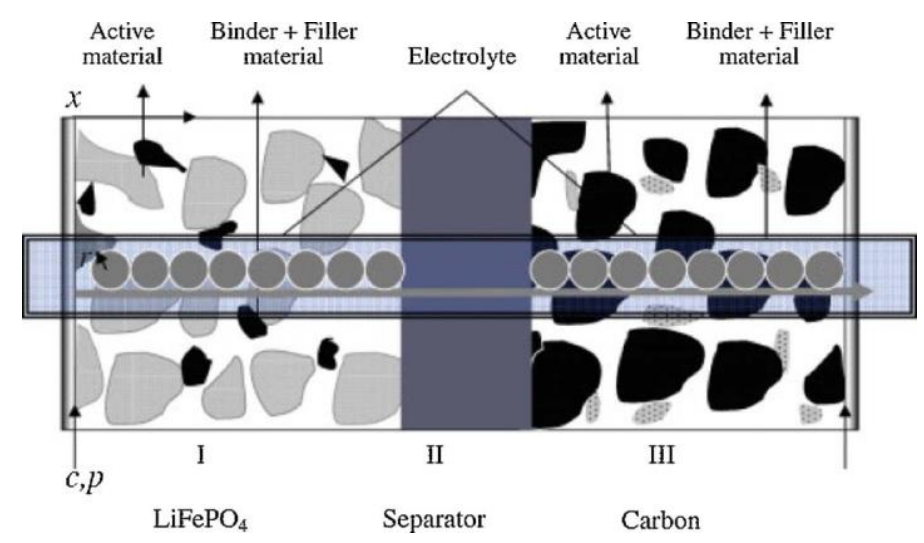

Figure 3. Schematic of the electrochemical model.

\subsection{Preventing thermal runaway using separators.}

The separator is one of the essential components of a $\mathrm{Li}$-ion battery. The separator may be manufactured from paper, gel, or chemical compound. Among these materials, the polymer separator is that the most generally utilised in commercial Liion batteries. As referenced, the separator is about within the positive and negative cathodes inside a battery. From the battery activity purpose of view, a separator serves two capacities: it forestalls direct contact between the 2 anodes, and it offers a pathway to the lithium ions. The separator is continually made of protection materials that won't cause an inside short out in the battery, and it features a porous construction which will ingest fluid electrolyte. Battery chemistry responses rely on this ionic pathway given by the extractor. The execution of a polymer separator likewise further develops Li-ion battery safety. Since a polymer separator has a permeable construction, if the battery temperature increments to approach the separator dissolving point, the separator pores will close. This pore conclusion measure is alluded to as "separator shutdown". The separator closure impedes the pathway between the positive and negative anodes, and stops the electrochemical responses. The battery resistance will likewise generally increment throughout this cycle. In the closure interaction, the chemical compound extractor won't give at once, which suggests that the separator closure won't utterly stop everything of the responses right away. As an on the spot outcome, the battery won't begin to relax off quickly throughout the separator closure or perhaps when the separator closure. The separator must hold its integrity during the closure cycle. one thing else, if the separator shrivels during the closure interaction, the hindered positive and negative terminals can foursquare interface with each other and result in interior short out. At this stage, the top side of the separator closure doesn't exist anymore. 2.3 fire anticipation using flame retardants within the thermal runaway cycle, the warmth delivering response can prompt the battery temperature increment, and also the gases collected within the battery will create the inward pressure increment. To any develop the battery safety and forestall an explosion, varied safety mechanisms are carried out. some techniques to figure on the thermal stability of the electrolyte have been proposed. One methodology is to feature flame retardants (FRs) to the electrolyte. The first necessity of the metal is that its flash points got to be higher than the comparison upsides of the acyclic solvents. In addition, the meted out FRs got to be latent and ought not impede the battery' presentation and also the chemistry responses within it. Triethyl phosphate (TEP) and Trimethyl phosphate (TMP) were shaky on the plumbago cathode. Hexamethylcyclophosphate (HMPN) features a high softening point, but the thickness is likewise high. Adding these FRs will subvert the battery' performance.[Found by Xu at al] As an additional substance to the electrolyte, to figure on the thermal stability of the electrolyte while not obstructing the sport execution, the load level of the flame retardant content is likewise unremarkably underneath 20\%. Quercetin, a replacement electrolyte additive, could be a polyphenolic three-ringed flavonoid compound with 5 phenolic resin hydroxyl group teams that possesses inhibitor properties commonly wont to defend food, prescribed drugs associated cosmetics from spoilage. Its chemical structure is shown in theme 1 . Thus, we have a tendency to were fascinated by investigation a doubtless similar 'protective' role for "quercetin" as an electrolyte additive in lithium-ion cells. The aim of this study was to look at the result of quercetin as an additive on the cell's chemistry performance with reference to charging/discharging, impedance, cycle life and overcharging safety. Instead of adding metals to the electrolyte directly, another strategy is to include the FRs in to the battery by confining them to the extractor. The FRs within the separator is intended to be discharged at an explicit temperature. The 
separator is formed of electro spun microfiber that's specially designed with a core-shell structure. The core is made of triphenyl phosphate (TPP), that is an FR. on the far side adding FR to the electrolyte, another strategy to extend battery safety is to switch this low flash purpose solvents with a high flash point electrolyte, which may create the electrolyte per se inert to fire.

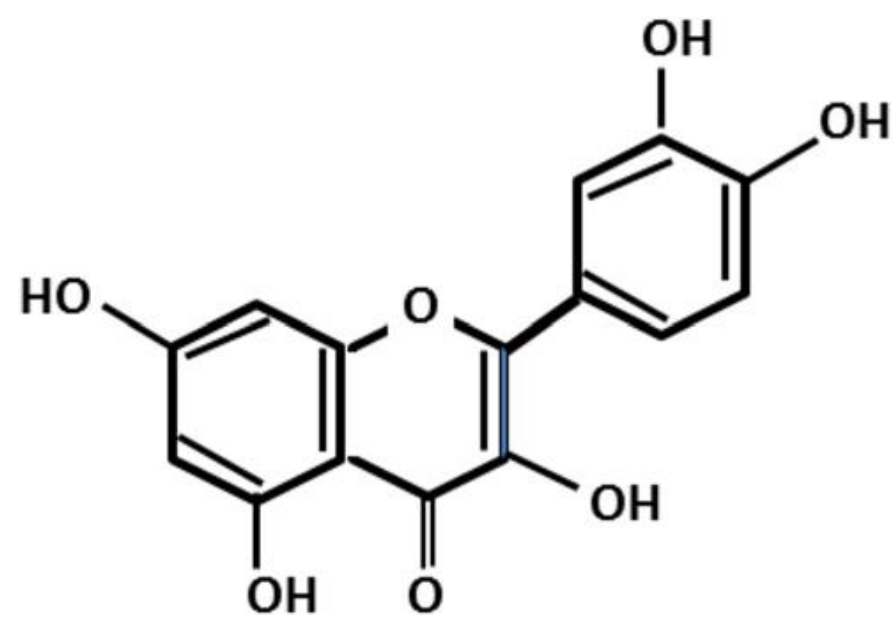

Scheme 1. Chemical structure of quercetin.

\subsection{Fire prevention using cell venting}

Another procedure for more developing battery safety is to feature safeguard instruments into the battery. frequently carried out safeguard elements incorporate safety vents, thermal fuses, and closedown centrifuges. Safety vents are intended to deliver additional inside pressure and forestall the nonstop increment of inner temperature. once the temperature is past the separator closure operating temperature range, the thermal runaway will proceed. Right now, flammable gases from the battery materials, together with natural electrolyte, can collect inside the battery. allowing the gas collection could induce an explosion of the battery. A cell-venting instrument is meant to decrease the battery' inside pressure and deliver the gases. At the purpose once the cell emission is initiated, the gathered gases within A battery can blast out at once. With the protection vents, the battery interior gases are delivered in a exceedingly controlled manner rather than in an uncontrolled explosion. when the venting, not completely will the assembled flammable gases be delivered, but a great deal of warmth contained within the gases can likewise be delivered to the atmosphere. Moreover, venting can diminish the pressure applied on the battery centrifuge and reduce the danger of battery interior short out. the concept of a battery vent intends to diminish the pressure developed within the thermal runaway method and forestall battery burst.

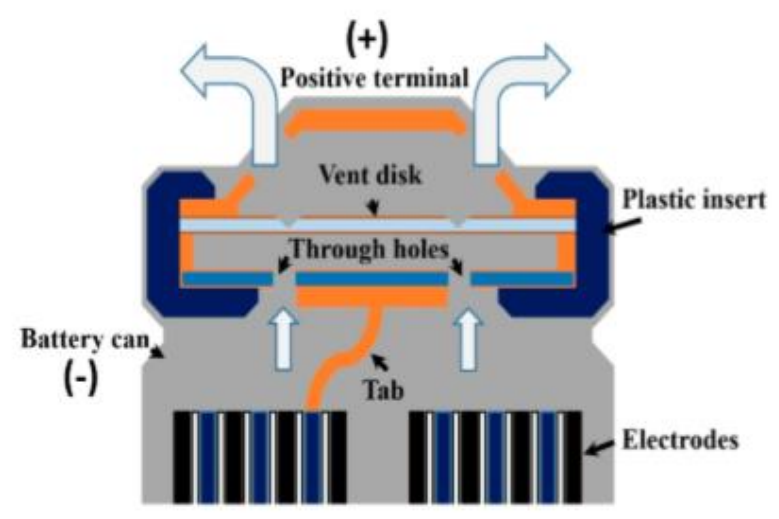

Figure 3. Typical structure of the battery cap

Table 1. Illustrates various classification of hazards in LIION batteries along with their description and hazard level [European Council of Research and Development]

\begin{tabular}{ccc}
\hline Hazard Level & Description & Classification Criteria and Effect \\
\hline 0 & No effect & No effect. No loss of functionality. \\
\hline 1 & Passive protection activated & $\begin{array}{c}\text { No defect; no leakage; no venting, fire, or flame; } \\
\text { no rupture; no explosion; no exothermic reaction } \\
\text { or thermal runaway. Cell reversibly damaged. } \\
\text { Repair of protection device needed. }\end{array}$ \\
\hline 2 & Defect/damage & $\begin{array}{c}\text { No leakage; no venting, fire, or flame; no rupture; } \\
\text { no explosion; no exothermic reaction or thermal } \\
\text { runaway. Cell irreversibly damaged. Repair } \\
\text { needed. }\end{array}$ \\
\hline 3 & $\begin{array}{c}\text { Leakage } \\
\Delta \text { mass }<50 \%\end{array}$ & $\begin{array}{c}\text { No venting, fire, or flame* no rupture; no } \\
\text { explosion. Weight loss }<50 \% \text { of electrolyte weight } \\
\text { (electrolyte }=\text { solvent + salt). }\end{array}$ \\
\hline 5 & $\begin{array}{c}\text { Venting } \\
\Delta \text { mass } \geq 50 \%\end{array}$ & $\begin{array}{c}\text { No fire or flame; no rupture; no explosion. } \\
\text { Weight loss } \geq 50 \% \text { of electrolyte weight } \\
\text { (electrolyte = solvent + salt). }\end{array}$ \\
\hline 6 & Fire or flame & No rupture; no explosion (i.e,, no flying parts). \\
\hline 7 & Rupture & No explosion, but flying parts of the active mass. \\
\hline Explosion & Explosion (i.e., disintegration of the cell). \\
\hline
\end{tabular}

\subsection{Extinguishing Li-ion battery fires}

The classification of a Li-ion battery fire will shift, nonetheless by and enormous fits into categories A, B, or C. Sometimes, a Li-ion battery is employed because the power source, and also the fire includes electrical gadgets. in numerous cases, a fire caused by a Li-ion battery can unfold and misstep available materials. fire dousers for Li-ion batteries fluctuate obsessed with the smothering specialist, like dry artificial compounds, carbon dioxide, froth, water, halons, and dry powders. CO2 will be utilized to stifle the fire, yet it doesn't chill the battery off. putting out a Li-ion battery fire alludes to each extinction the open flame and decreasing the battery temperature. Current norms don't have specific requirements for dousing Li-ion battery fires. Principles for Li-ion batteries are distributed by the Institute of Electrical and electronics Engineers (IEEE), Underwriters Laboratories (UL), and also the united nations (UN), but they center simply around battery misuse testing to ensure that $\mathrm{Li}$-ion batteries are protected throughout transportation. The tests are meant to be directed beneath harmful conditions, like break, drop, shock, warming, and 
weird charging. This exploration hole constrained the NFPA to guide an analysis of Li-ion battery perils. The identified problems known with stifling Li-ion battery fires are: restricted comprehension of battery flammability, absence of fire assurance specifications for battery packs, and hazy adequacy of potential fire-quenching suppressants.

\subsection{Simple tips for using Lithium-ion Batteries}

- A failing Li-ion begins to hiss, bulge and leak electrolyte.

- The electrolyte consists of lithium salt in an organic solvent (lithium hexafluorophosphate) and is very flammable. Burning electrolyte will ignite fuel in shut proximity.

- Dowse Li-ion fire with water or use a daily extinguisher. solely use a category D fire extinguisher for lithium-metal fires because of the reaction of water with metal. (Li-ion contains very little lithium metal reacting with water.)

- If a category D asphyxiator isn't available, douse a lithiummetal fire with water to stop the hearth from spreading.

- For best results dowse a Li-ion fire, use a foam extinguisher, $\mathrm{CO} 2$, abc dry chemical, fine graphite, copper powder or soda (sodium carbonate) as you'd extinguish alternative flamable fires. Reserve the category D extinguishers for lithium-metal fires only.

- If the fire of a burning lithium-ion battery can not be extinguished, permit the pack to burn in an exceedingly controlled and safe way.

- bear in mind of cell propagation as every cell may well be consumed on its own plan once hot. Place a apparently burned-out pack outside for a time.

\section{QUERCETIN}

\subsection{Quercetin as an electrolytic additive}

Quercetin, a new electrolyte additive, could be a polyphenolic three-ringed flavonoid compound with 5 phenoplast hydroxyl group teams that possesses inhibitor properties unremarkably used to shield food, prescription drugs and cosmetics from spoilage. Its chemical structure is shown in scheme 1. Thus, we were inquisitive about work a probably similar 'protective' role for "quercetin" as an electrolyte additive in lithium-ion cells. The aim of this study was to look at the result of quercetin as an additive on the cell's electrochemical performance with regard to charging/discharging, impedance, cycle life and overcharging safety. In a trial to ameliorate the poor cyclability of Li-ion at elevated temperature, quercetin is applied as an additive. The improved cyclability of the quercetin-containing cell at high temperature implies that by forming strong and less-resistive SEI, quercetin is preferentially oxidized and passivates the Li-ion electrode.EIS result coherently suggests that the quercetin-added electrolyte forms a more compact and Li-ion conducting interface. Thepresence of quercetin restrains the formation of $\mathrm{LiF}$, suppresses the reaction of PF5, and alleviates Mn dissolution. Quercetin will resultively prolong the cycle-life of Li-ion at elevated temperature. Its potential and sensible usage as an electrolyte additive for high-voltage cathode. The effect of quercetin as an electrolyte additive for high-voltage Li-ion battery was investigated at elevated temperature. By potential sweep analysis, quercetin was discovered to preferentially oxidize and irreversibly form SEI layer on Li-ion battery. At high temperature, Li-ion battery (quercetin employed) electrolyte achieved improved cycle retention compared to quercetin-free electrolyte, retentive $89 \%$ of its initial discharge capacity. By virtue of the passivating and scavengingability of quercetin, the gratuitous electrolyte decomposition and metal dissolution were mitigated. Consequently, within the presence of quercetin, Li-ion battery might maintain its charged state beneath high voltage, indicating that quercetin may well be employed as an electrolyte additive for high-voltage cathode for Lithium-ion batteries at elevated temperature. the safety and therefore the cycle lifetime of the lithium-ion batteries were considerably improved by using quercetin, Associate in Nursing organic flavonoid compound, that could be a polyphenolic inhibitor utilized in combustibles, as an electrolyte additive. Quercetin $(0.05 \%)$ containing electrolytes exhibited better cycling ability than did the electrolytes while not quercetin. the improved cycling performance is attributed to the resistivity suppression capability of quercetin throughout prolonged cycles of charging and discharging. The electrolyte with quercetin exhibits overcharge tolerance and thermal stability whereas the lithium-ion battery undergoes overcharging, so increasing the safety of batteries by minimizing the chance of fire and explosion. The enhanced performance of the quercetin-contained solution may well be due to the thin passivation microstructure on the cathode's surface fashioned by the polyphenolic compound that results in the inner resistivity suppression throughout cycling. Quercetin, already well-known to own a protecting perform as an inhibitor in physiological systems, might become a extremely promising candidate as an electrolyte additive conferring similar protection to reversible lithium-ion batteries.

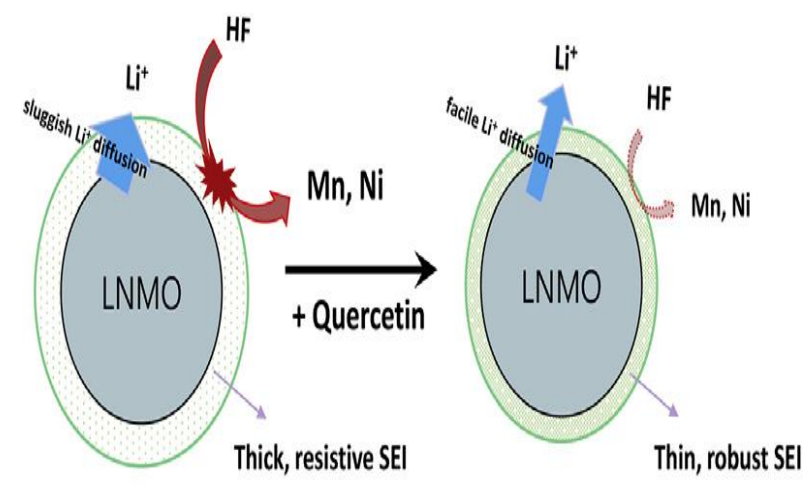

blank

07.5 


\section{Figure 4}

\section{Cell venting}

The gases released from a Li-ion battery cell may be toxic, e.g., $\mathrm{CO}$, but the fluoride emissions are of maximum concern. Hydrogen fluoride (HF) is one in every of them, however there also are others, e.g., phosphorousoxyfluoride (POF3). They are formed from the fluorine content used withinside the Li-ion cell. One of the passive protection additives in a lithium ion cell is the cell-can's mechanical protection vent, that's meant to launch the inner stress of the cell whilst a distinct stress is reached, however prismatic cells swell and cylindrical cells bulge in the course of pressurization. It is therefore, acceptable to check the cell's venting mechanism in a managed way to be able to make certain that each the cell's can and the overall performance of the protection vent isn't always compromised. Safety vent is critical to shield its users from unpredictable explosions as a result of the growing inner stress of lithium-ion batteries. In order to save you the explosion of the battery, a protection vent rupture is needed whilst the inner stress reaches a essential value. In traditional production, the cap plate and protection vent are fabricated one by one and are then welded. During the cycles of lithium-ion battery, it super venesa diversity of chemical and thermal reactions below overcharging and overheating. For this reason, the cells can generate gases and growth the inner stress. In severe cases, the battery can result in an explosion and combustion. In order to save you the explosion of the battery, protection gadgets are required in every cell, together with shutdown separator, tearaway tab, protection vent, and thermal interrupt [9]. In particular, protection vents save you explosions withinside the lithium-ion battery, and play a prime position in securing the user's protection via way of means of rupturing in advance than different elements whilst a sure internal stress degree is reached. Therefore, it is critical to predict the rupture stress of protection vents. In the traditional production technique, the cap plate and protection vent are one by one fabricated and, then, welded together, as proven in Fig. 5(a). The welded protection vent can also additionally have low product reliability and productiveness because of the welding defects and complicated strategies. This have a look at offers the producing technique of the incorporated protection vent to growth the performance of productiveness. As indicated in Fig. 2, the incorporated protection vent is synthetic via way of means of the backward extrusion and coining technique. After the backward extrusion and coining strategies have been completed, the incorporated protection vent is annealed to put off the residual strain as a result of the forming technique. The incorporated protection vent, which became fabricated via those production strategies, had the operation mechanism as proven in Fig. 6. The inner stress happens withinside the prismaticlithium-ion battery because of the lengthy intervals of operation

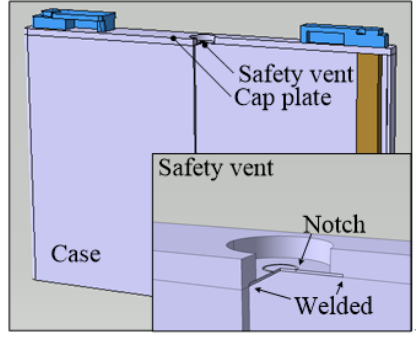

(a) Welded safety vent

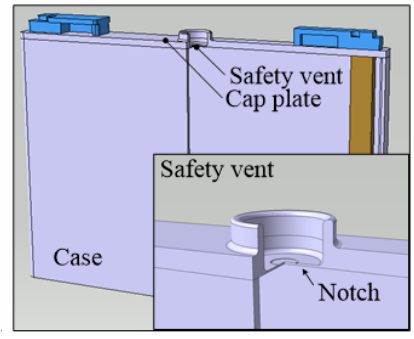

(b) Integrated safety vent
Figure 5. Comparison of welded safety vent with integrated safety vent.

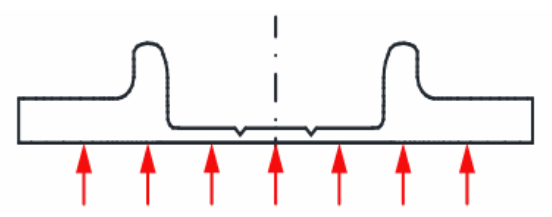

(a) Occurrence of internal pressure

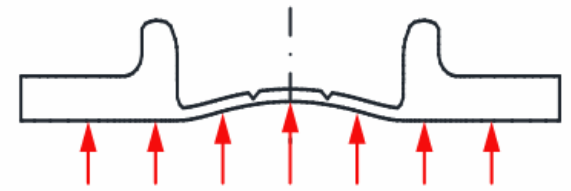

(b) Increase of internal pressure

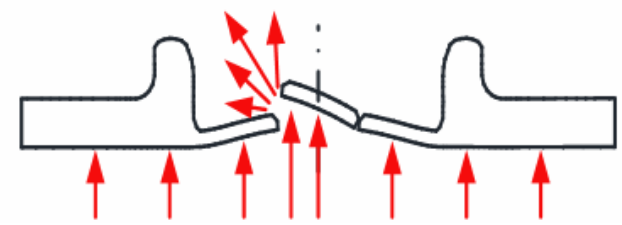

(c) Rupture of safety vent and leakage of pressure

Figure 6. Operation mechanism of the integrated safety vent.

$$
\text { V. EXPERIMENT }
$$

\subsection{SAMPLES}

Two styles of commercial LIBs with varied cathode materials were utilised during this work. They were made by Linde and Hyundai, individually, and their anode materials were comparable, let's say graphite. The cathode material for the previous was NMC, whereas that for the latter was LFO. before tests, the primary energy put away in batteries was delivered by cathartic with a consistent current (CC) till the voltage fell upon a selected value (the discharge cut off voltage), and after the batteries would be charged by an identical CC to the fixed voltage. Henceforth, the batteries were saved still for $24 \mathrm{~h}$ to ensure they stayed stable before tests. 


\subsection{APPARATUSES}

As displayed in Figure 2. tests were completed in a very much ventilated chamber. The battery was set upon a supporting cross section made of iron wire. The electric warmer was situated underneath the lattice a ways off of $1 \mathrm{~m}$ from the battery. A K-type thermocouple was appended to the upper surface of the battery to quantify its surface temperature and the temperature was recorded at the information obtaining gear by a PC. The other four thermocouples were set up around the battery to gauge the fire temperature. Cycling battery was accomplished by charge/release cycle gear. At long last, a radiometer sensor $2 \mathrm{~m}$ on a level plane away from the battery to gauge the warmth transition of fire and it was put confronting the safety vent with the goal that the best warmth motion could be procured when LIBs burst into flames.

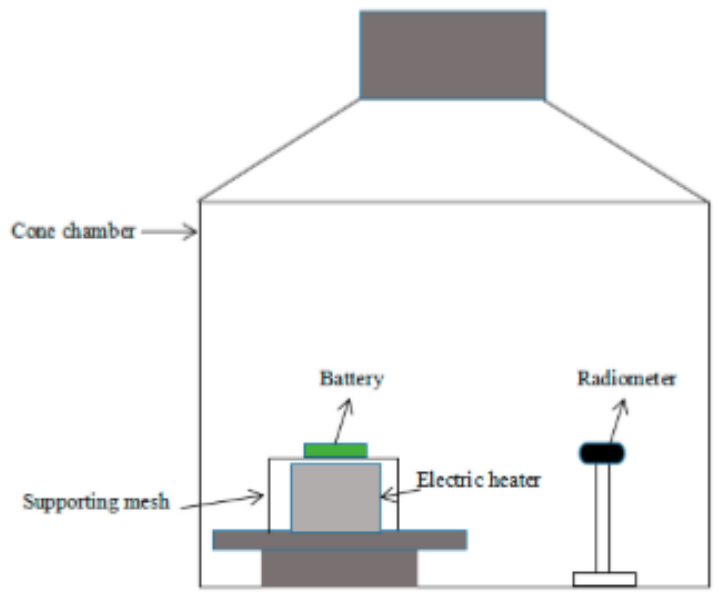

Figure 2. Schematic of Experimental Setup

\subsection{RESULTS AND DISCUSSIONS}

\subsubsection{Thermal Behaviors of LIB during Charging and Discharging}

The batteries were discharged at a rate to the discharging cutoff voltage $(25 \mathrm{~V})$. It was seen that the releasing interaction of LIB can be isolated into three sections. At the underlying portion of releasing, there exists a short stage where voltage drops forcefully. With the increment of voltage, the stage will be more self-evident and last more. The length of this portion is short. From that point onward, the battery enters a stable releasing section where voltage diminishes at a somewhat steady rate until the battery gets into the epilog of releasing. At the third section, the voltage decreases pointedly. Before long, the releasing finishes when the voltage falls. It is tracked down that the underlying releasing voltage increments with the development of remove voltage.

\subsubsection{Burning Process}

During the warming stage, the battery stayed steady and just piece of the pressing dissolved. With the nonstop ascent of temperature, the safety vent broke, joined by an unmistakable sound. In the future, smoke was delivered and afterward the smoke touched off. The flame went on until an enormous amount of smoke was freed, bringing about the savage discharge and afterward stable burning could be observed.This reveals that the overcharged LIB possesses a more serious ignition measure and a lower dependability than the ordinary LIB. It is the aftereffect of shaky electroactive materials, where profoundly delithiated electroactive materials become more responsive in the cheated LIB.

\subsubsection{Surface Temperature}

At the warming stage, the surface temperature rises easily because of ceaseless outside warming, and the pressing of the battery softens gradually. Subsequently, it tends to be seen that the safety vent breaks were joined by some smoke ejected. The smoke catapulted is lighted later and a fire shows up, trailed by a sped up temperature rise. Hereafter, thermal runaway happens and it very well may be seen that the temperature increments forcefully to a peak. Meanwhile, with the expanding voltage, the fire arises prior and the greatest surface temperature has an upturn. In particular, a LIB overcharged past the cut off voltage is more inclined to be temperamental and let completely go. The wonder will turn out to be more terrible for a profoundly overcharged LIB.

\subsubsection{Relative Heat Flux}

Radiative heat flux results from the temperature contrast between two surfaces. Fundamentally, the radiative heat flux is equivalent to the pattern of the consuming rate. More brutal consuming has a higher radiative heat flux. In addition, the all out radiative heat flux and absolute radiative heat likewise introduced a comparative wonder. This demonstrates that the NMC with higher voltage will deliver more warmth subsequent to bursting into flames. Besides, it was tracked down that the pinnacle heat transition of NMC with higher voltage will show up prior. Then again, LFP batteries display comparable conditions in that the pinnacle heat motion and all out radiative warmth develop with the increment in voltage. This is credited to the rough launch of a profoundly overcharged battery, bringing about inadequate ignition and less warmth being delivered. This can be affirmed by the overall perceptions ablaze conduct.

\subsubsection{Discussion}

The trial results uncover that a battery overcharged past the discharge voltage will display higher fire danger, and the peril increments with the ascent in voltage. To clarify why overcharging extraordinarily influences the warm practices of LIB, the charging instrument of LIB should be explained. At the point when a LIB is in a condition of harmony, there will be a lithium ion focus contrast between the cathode and anode when battery is completely energized. During charging, a 
voltage applied across the terminals powers lithium ions to be extricated from the cathode precious stone and moved to the graphite anode through electrolyte. This outcomes in the decrease in the lithium ion fixation in the cathode and development in the anode.

\section{CONCLUSION}

To have a superior comprehension of the fire perils of overcharged LIB, two broadly utilized commercial LIBs, NMC and LFP, with various discharge voltage, were tried in this work. The definite examination of the consuming marvels, the surface temperature, the fire temperature, voltage, and radiative heat flux offered here addresses an exhaustive comprehension of the fire perils presented by burning LIB.

Various Li-ion battery fire and blast episodes have drawn in increasingly more regard for the issue of battery wellbeing. This paper talked about three systems for lessening the quantity of battery fire episodes: separator closure, flame retardants, and cell venting. Albeit the most generally utilized polymer separator has a closure work, when the separator contracts and loses its trustworthiness, the closure work is futile. With respect to flame retardants, the significant issue is whether they upset the battery's exhibition. Late exploration showed the chance of supplanting the carbonate-based electrolyte with another organic electrolyte that has better thermal stability. The execution of cell venting can't ensure that the warmed battery content will be obstructed inside the battery case during the warm rampant.

Safety issues identified with thermal runaway is an extremely convoluted interaction including science, material science and designing, and it ought to be considered from terminal materials and electrolyte to cell plan. The overall warm out of control hypothesis was proposed and it is clear. In any case, various terminal materials, electrolytes and cell types show distinctive thermal runaway practices, and these careful responses at various thermal runaway stages is under examining, which are relying upon the part materials, cathode, anode and electrolyte. The responses additionally are overwhelmed by the condition of charge, releasing rate, and so on To reveal these responses need our supporting exploration.

The impact of quercetin as an electrolyte added substance for high-voltage Li-ion battery as researched at raised temperature. By potential scope examination, quercetin was uncovered to specially oxidize and irreversibly structure SEI layer on Li-ion battery. At high temperature, Li-ion in quercetin utilized electrolyte accomplished further developed cycle maintenance contrasted with sans quercetin electrolyte, holding $89 \%$ of its underlying release limit. By excellence of the passivating and scavengingability of quercetin, the superfluous electrolyte deterioration and metal disintegration were relieved.

The assembling interaction of the coordinated safety vent was recommended to improve the proficiency and usefulness as contrasted and the welded safety vent. The assembling interaction of the coordinated safety vent incorporates the retrogressive expulsion measure and begetting measure. The safety vent was planned in a shape that can prompt explosion under the necessary pressure.

The battery venting configuration ought to consider impeding the warmed battery content while delivering the collected gases. Bringing down the gas discharge edge or including a screen the vent can further develop the venting capacity. The methodologies for stifling Li-ion battery fires were likewise talked about. Flow accessible guidelines give battery security testing under oppressive conditions, including electrical/mechanical/warm maltreatment, however don't give direction to battery fire assurance or dousing Li-ion battery fires. When stifling battery fires, cooling the battery is pretty much as significant as smothering the flame.

\section{REFERENCES}

1. Wang, Q.; Sun, J.; Yao, X.; Chen, C. Thermal Behavior of Lithiated Graphite with Electrolyte in Lithium-Ion Batteries. J. Electrochem. Soc. 2006, 153, A329-A333.

2. Al Hallaj, S.; Maleki, H.; Hong, J.; Selman, J. Thermal modeling and design considerations of lithium-ion batteries. J. Power Sources 1999, 83, 1-8.

3. Feng, X.; Fang, M.; He, X.; Ouyang, M.; Lu, L.; Wang, H.; Zhang, M. Thermal runaway features of large format prismatic lithium ion battery using extended volume accelerating rate calorimetry. J. Power Sources 2014, 255, 294-301.

4. Lee, C.W.; Venkatachalapathy, R.; Prakash, J.A. novel flame-retardant additive for lithium batteries. Electrochem. Solid-State Lett. 2000, 3, 63-65.

5. Xiang, H.; Xu, H.; Wang, Z.; Chen, C. Dimethyl methylphosphonate (DMMP) as an efficient flame retardant additive for the lithium-ion battery electrolytes. J. Power Sources 2007, 173, 562-564.

6. Yao, X.; Xie, S.; Chen, C.; Wang, Q.; Sun, J.; Li, Y.; Lu, S. Comparative study of trimethyl phosphite and trimethyl phosphate as electrolyte additives in lithium ion batteries. J. Power Sources 2005, 144, 170-175. 
7. Shim, E.G.; Nam, T.H.; Kim, J.G.; Kim, H.S.; Moon, S.I. Electrochemical performance of lithium-ion batteries with triphenylphosphate as a flame-retardant additive. J. Power Sources 2007, 172, 919-924.

8. Josefowitz, W.; Kranz, H.; Macerata, D.; Soczka-Guth, T.; Mettlach, H.; Porcellato, D.; Orsini, F.; Hansson, J. Assessment and Testing of Advanced Energy Storage Systems for Propulsion-European Testing Report. InProceedingsofthe21stWorldwideBattery, Hybrid and Fuel Cell Electric Vehicle Symposium \& Exhibition, Monte Carlo, Monaco, 2-6 April 2005.

9. National Fire Protection Association. Portable Fire Extinguishers; National Fire Protection Association: Quincy, MA, USA, 2013. 